\title{
The interaction between time and number in a temporal bisection task: A reply to Vicario (2011)
}

\author{
Massimo Grassi, Mario Bonato \\ Dipartimento di Psicologia Generale, Università di Padova, via Venezia 8, I 35131 Padua, Italy; \\ e-mail: massimo.grassi@unipd.it \\ Received 20 July 2011, in revised form 19 April 2012
}

\begin{abstract}
We discuss the results of Vicario (2011, Perception 40 23-29), in the light of an experiment designed to bypass some of the limits of that study. There, participants were asked to perform a temporal bisection on numerical stimuli (small or large digits) presented either for $700 / 900 \mathrm{~ms}$ or $2000 / 2200 \mathrm{~ms}$. For the two longest durations only, bisections of larger digits occurred later than those of smaller digits. Here, subjects judged the temporal position of a flick occurring during the visual presentation of a digit $(1,5$, or 9) which lasted on the screen for either $700 \mathrm{~ms}$ or $2000 \mathrm{~ms}$. Results revealed no difference in the perceived temporal midpoints of large compared to small digits. In contrast, they showed a response bias: only with the shortest-duration stimuli the digit's magnitude affected the subject's response.
\end{abstract}

Keywords: time, bisection, number magnitude

Vicario (2011) recently reported an effect of numerical magnitude in a temporal bisection task. He asked participants to monitor a digit presented at the centre of the screen for a variable duration $(700 / 900 \mathrm{~ms}$ or $2000 / 2200 \mathrm{~ms})$. The participant was asked to imagine a timer starting after the disappearance of the digit and to stop the timer, by pressing a response key, when she/he felt that half of the digit's duration had elapsed. In experiment 1 digits $1-2$ and $8-9$ were presented in separate blocks and no magnituderelated effect emerged. In experiment 2 , the same digits were presented within a single block of trials and, for the two longest durations only, larger numbers induced bisections later in time than smaller numbers.

The result reported by Vicario deserves attention. However, the method he adopted has several constraints. First, Vicario's approach is inadequate for estimating the effect of the number magnitude at short durations. In Vicario's experiment, the temporal bisections of 700-ms-long stimuli were greater than $700 \mathrm{~ms}$, ie the bisections laid outside the stimulus's duration. As the author himself remarks (page 27) "the absence of time-numerosity interaction in the subsecond block could be explained in terms of data concerning the participants' accuracy". Second, Vicario discusses the result as an effect of numerical magnitude on time perception. However, in his task, responses could not be classified as correct or incorrect. Thus, it is impossible to disentangle whether the effect observed reflects a genuine perceptual effect, a response bias, or both. Here, we assessed the possibility to reproduce Vicario's findings with a different method designed to bypass these methodological issues.

Eleven subjects viewed a black fixation point $(0.3 \mathrm{deg})$ followed after $1000 \mathrm{~ms}$ by a black digit (Arial font, $1.0 \mathrm{deg}$ ) presented at the centre of a white screen. Digits were either 1,5 , or 9 and lasted on the screen for either $700 \mathrm{~ms}$ or $2000 \mathrm{~ms}$. In each trial one frame of the digit $(10 \mathrm{~ms})$ was coloured in white producing a 'flick' in the stimulus perception. The flick was positioned at either $\pm 7 \%, \pm 21 \%, \pm 36 \%$ with respect to the digit's temporal centre. The subject reported to the experimenter (who sat behind a screen) whether the flick occurred in the first or in the second half of the digit's duration. This task transposes in the temporal domain the landmark task, a variant of 
the bisection task widely adopted to avoid motor confounds. There were 15 trials for each condition within a single block of random trials.

To estimate the subjects' psychometric functions for each digit and duration, logistic regressions were performed on individual proportions of "flick in the second half" responses. The midpoints of psychometric functions are the subjective temporal midpoints of the stimuli and are presented, averaged across subjects, in the top part of the figure. Successively, we classified responses between hits (ie response "flick in the second half" for flick in the second half) and false alarms (ie response "flick in the second half" for flick in the first half). We then calculated the $d^{\prime}$ and $c$ indexes of the signal detection theory and calculated 2 (duration) by 3 (digit) two-way ANOVAs. The $d^{\prime}$ ANOVA revealed a better performance for $2000 \mathrm{~ms}$ than for $700 \mathrm{~ms}$ stimuli $\left(F_{1,10}=87.56\right.$, $\left.p<0.0001, \eta_{\mathrm{p}}^{2}=0.89\right)\left(d_{2000}^{\prime}=2.41 \pm 0.66\right.$ and $\left.d_{700}^{\prime}=1.44 \pm 0.66\right)$, whereas the factors "digit" and the interaction were not significant (both $F \mathrm{~s}<1$ ). In contrast, the $c$ ANOVA revealed no effect of duration $\left(F_{1,10}=3.35, p=0.097, \eta_{\mathrm{p}}^{2}=0.25\right)$, but an effect of the digit's magnitude $\left(F_{2,20}=3.75, p<0.041, \eta_{\mathrm{p}}^{2}=0.27\right)$. Crucially, an interaction emerged between duration and digit's magnitude $\left(F_{2,20}=4.43, p<0.026\right.$, $\eta_{\mathrm{p}}^{2}=0.31$ ): for the $700 \mathrm{~ms}$ stimuli only, subjects were more prone to respond "flick in the second position" the larger the digit's magnitude.

The current study investigated the effect of numerical magnitude on temporal bisection. In contrast to the method proposed by Vicario (2011), the method we adopted
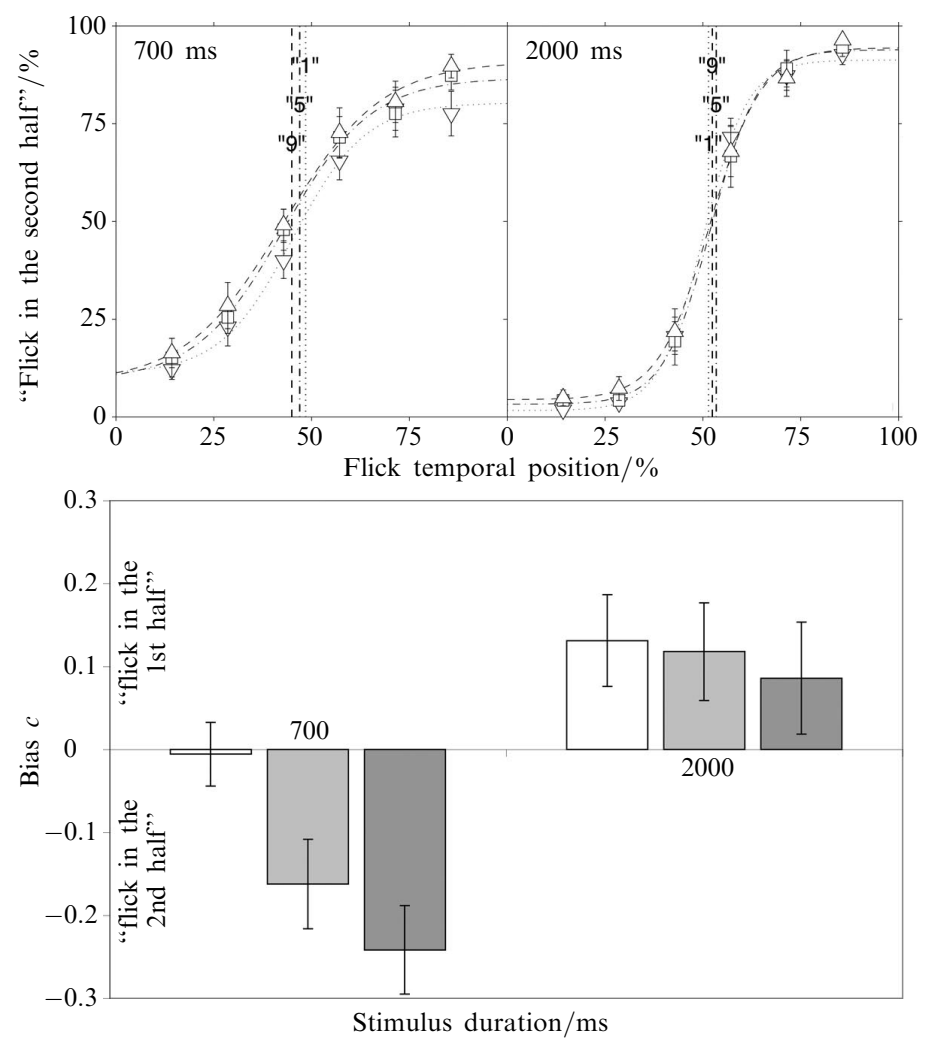

Figure 1. Top graph: responses "flick in the second half" as a function of the flick's temporal position. Pointing-down triangles: responses for digit "1"; squares: digit "5"; pointing-up triangles: digit "9". Dotted "1", dash-dotted ("5"), and dashed ("9") sigmoid curves are logistic interpolations of subjects' responses. Vertical lines represent the mean subjective (interpolated) temporal midpoints. Bottom graph: response bias as a function of stimulus duration. In all graphs, error bars represent $\pm 1 \mathrm{SE}$. 
is suitable to investigate also brief durations, as indexed by temporal midpoints which laid within the duration of the stimuli. In addition, it also allows to disentangle stimulus-based from response-based effects. In this respect, our results are different from those observed by Vicario (2011). The $d^{\prime}$ analysis did not reveal perceptual differences between the temporal bisections of large and small digits either with $700 \mathrm{~ms}$ or with $2000 \mathrm{~ms}$ stimuli. In contrast, we observed a response bias for the shortest-duration stimuli where subjects responded more often "flick in the first half" for small magnitudes and "flick in the second half" for large magnitudes. This bias plausibly resulted from a coupling between numerical magnitude and temporally characterised responses: for example, " 1 " was associated (eg either spatially or conceptually) with "first half" whereas "9" was associated with "second half".

The processing of short and long durations is thought to be underpinned by different cognitive mechanisms (see Grondin 2010 for a review). Long (but not short) durations allow the implementation of several alternative processing strategies (eg counting). It is thus possible that the heterogeneity of these strategies might attenuate the effect of numerical magnitude upon response selection, thus causing the effect to merge only at the shortest duration. Alternatively (or in addition) it is plausible that the representation of number's magnitude decays rapidly when the number is task-irrelevant (as in the current experiment). This might explain why the numerical magnitude had an effect only at the shortest (and not at the longest) duration. The current results add further evidence for an integration between time and number magnitude (eg Walsh 2003) and, above all, question to what extent the effect observed by Vicario (2011) might be reliably extended to a different paradigm.

Acknowledgments. We would like to thank Maria Cristina Manuello for helping with the data collection. We are also grateful for comments on an earlier version of the paper by Simon Grondin and an anonymous reviewer.

\section{References}

Grondin S, 2010 "Timing and time perception: A review of recent behavioral and neuroscience findings and theoretical directions" Attention, Perception \& Psychophysics $72561-582$

Vicario C M, 2011 "Perceiving numbers affects the subjective temporal midpoint" Perception 40 $23-29$

Walsh V, 2003 "A theory of magnitude: common cortical metrics of time, space, and quantity" Trends in Cognitive Sciences 7 483-488 
Conditions of use. This article may be downloaded from the E\&P website for personal research by members of subscribing organisations. This PDF may not be placed on any website (or other online distribution system) without permission of the publisher. 